\title{
HUMANIZAÇÃO NO PROCESSO DE TRABALHO NA PERCEPÇÃO DE ENFERMEIROS DE UNIDADE DE TERAPIA INTENSIVA
}

\author{
Júlia Trevisan Martins ${ }^{1}$, Maria José Quina Galdino ${ }^{2}$, Mara Lúcia Garanhani ${ }^{3}$, Keity Mayumi Sammi ${ }^{4}$, Gabriela Schmitt Trevisan $^{5}$ \\ ${ }^{1}$ Enfermeira. Doutora em Enfermagem. Docente do Departamento de Enfermagem da Universidade Estadual de Londrina. \\ Londrina, PR, Brasil. \\ ${ }^{2}$ Enfermeira. Mestranda em Enfermagem da Universidade Estadual de Londrina. Docente do Departamento de Enfermagem \\ da Universidade Estadual do Norte do Paraná. Bandeirantes, PR, Brasil. \\ ${ }^{3}$ Enfermeira. Doutora em Enfermagem. Docente do Departamento de Enfermagem da Universidade Estadual de Londrina. \\ Londrina, PR, Brasil. \\ ${ }^{4}$ Enfermeira. Santa Casa de Misericórdia. Londrina, PR, Brasil. \\ ${ }^{5}$ Graduanda em Medicina pela Universidade do Oeste Paulista. Presidente Prudente, SP, Brasil.
}

RESUMO: Objetivou-se identificar os fatores que propiciam e dificultam a humanização entre os trabalhadores de enfermagem, na percepção de enfermeiros de uma unidade de terapia intensiva. Pesquisa qualitativa-descritiva realizada em 2012, com 10 enfermeiros que atuavam em uma unidade de terapia intensiva de adultos de um hospital universitário paranaense. Os dados coletados por entrevista semiestruturada foram analisados segundo a análise de conteúdo. Emergiram duas categorias: fatores que propiciam e fatores que dificultam a humanização laboral entre os trabalhadores de enfermagem. Concluiu-se que os fatores contributivos para a humanização relacionavam-se ao trabalho realizado em equipe, ao bom relacionamento interpessoal, ao reconhecimento por colegas, pela instituição e pelo próprio trabalhador, à comunicação efetiva, e aos valores pessoais. Já a falta de reconhecimento pelo trabalho desenvolvido e as atitudes de individualismo foram fatores que dificultavam a concretização das relações, para se estabelecer a humanização entre os trabalhadores.

DESCRITORES: Humanização da assistência; Ambiente de trabalho; Enfermagem de cuidados críticos; Unidades de terapia intensiva.

\section{HUMANIZATION IN THE WORK PROCESS IN THE VIEW OF INTENSIVE CARE UNIT NURSES}

\begin{abstract}
This study aimed to identify the factors which promote and hinder humanization among nursing staff, in the perception of the nurses from an intensive care unit. This qualitative-descriptive study was undertaken in 2012, with 10 nurses who worked in an adult intensive care unit in a teaching hospital in the Brazilian state of Paraná. The data, collected through semistructured interviews, were analyzed using content analysis. Two categories emerged: factors which promote, and factors which hinder, workrelated humanization among the nursing workers. It was concluded that the factors which contributed to humanization were related to the work undertaken as a team, to good interpersonal relationships, to recognition by colleagues, the institution and the worker herself, to effective communication, and to personal values. Lack of recognition for the work undertaken, on the other hand, and attitudes of individualism, were factors which hindered the realizationof the relationships, for establishing humanization among the workers.
\end{abstract}

DESCRIPTORS: Humanization of care; Work environment; Critical care nursing; Intensive care units.

\section{HUMANIZACIÓN EN EL PROCESO DE TRABAJO EN LA PERCEPCIÓN DE ENFERMEROS DE UNIDAD DE TERAPIA INTENSIVA}

RESUMEN: La finalidad del estudio fue identificar los factores que propicían y los que dificultan la humanización entre los trabajadores de enfermería, en la percepción de enfermeros de una unidad de terapia intensiva. Investigación cualitativa descriptiva realizada en 2012, con 10 enfermeros de una unidad de terapia intensiva de adultos de hospital universitario de Paraná. Los datos obtenidos por entrevista semiestructurada fueron analizados de acuerdo al análisis de contenido. Resultaron dos categorías: factores que propicían y factores que dificultan la humanización laboral entre los trabajadores de enfermería. Se concluye que los factores contributivos para la humanización se asocían al trabajo realizado en equipo, la buena relación interpersonal, el reconocimiento por compañeros, por la institución y por el propio trabajador, la comunicación efectiva, y los valores personales. Por otra parte, la falta de reconocimiento por el trabajo desarrollado y las actitudes de individualismo fueron factores que dificultaron la concretización de las relaciones para que se estableciera la humanización entre los trabajadores.

DESCRIPTORES: Humanización de la asistencia; Ambiente de trabajo; Enfermería de cuidados críticos; Unidades de terapia intensiva.
Autor Correspondente:

Maria José Quina Galdino

Universidade Estadual do Norte do Paraná

Rodovia BR 369, km 54 - 86360-000 - Bandeirantes, PR, Brasil

E-mail: mariagaldino@uenp.edu.br
Recebido: 27/05/2015

Finalizado: 08/07/2015 


\section{INTRODUÇÃO}

O processo laborativo na área de enfermagem engloba vários elementos em um único processo, e o uso de diferentes tecnologias, saberes e práticas específicas. Entretanto, nessa área, predomina um processo organizativo e de gestão pautado no modelo taylorista ${ }^{(1)}$.

Historicamente, o cuidado caracteriza o trabalho da enfermagem. A gestão, porém, está pautada no paradigma centralizado, vertical e formal, com ênfase na fragmentação do trabalho e com tarefas detalhadas e inflexíveis voltadas a manuais de procedimentos e rotinas, normas, as quais, entre outros fatores, desvalorizam as relações interpessoais - entre os profissionais e entre estes e os usuários. Em consequência, o trabalhador não se apropria de seu processo de trabalho, dificultando-lhe o exercício da autonomia na execução das políticas e na produção de saúde, além de lhe acarretar desmotivação e insatisfação ${ }^{(1-2)}$.

Dessa forma, fica evidenciado que é necessário o envolvimento dos gestores, dos trabalhadores e dos pacientes para que se possam desenvolver práticas humanizadas, tanto voltadas para os pacientes quanto para as equipes de trabalho.

Entende-se por humanização a modificação de ordem cultural das práticas de atenção aos usuários e da gestão de processos de trabalho. Com essa compreensão em 2004, o Ministério da Saúde propôs a Política Nacional de Humanização (PNH), que se pauta no "fortalecimento de trabalho em equipe multiprofissional, fomentando a transversalidade e a grupalidade ${ }^{\prime(3: 8)}$. Assim, quando há trabalho em equipe envolto nas premissas da humanização, o indivíduo é considerado participante do seu cuidado, e os atos em saúde vão ao encontro de sua individualidade, visualizando a pessoa como um todo e não como parte de uma máquina em manutenção. Por essa razão, a humanização da atenção entre profissional e usuário é uma vertente bastante explorada nas pesquisas na área da saúde, principalmente na área da enfermagem ${ }^{(4)}$.

Entretanto, as condições de trabalho, a motivação e, consequentemente, o bemestar dos profissionais de saúde têm sido relegados ao segundo plano, ou completamente desconsiderados. É preciso, pois, que os gestores e profissionais criem espaços para valorizar o trabalhador e propiciem ambientes facilitadores de ações de humanização, para que os trabalhadores também possam ter as suas necessidades físicas e psicológicas satisfeitas ${ }^{(5)}$.

No contexto hospitalar, a Unidade de Terapia Intensiva (UTI) se caracteriza por um cenário de cuidado considerado altamente técnico, objetivo e contínuo, desenvolvido por uma equipe multidisciplinar (médicos, enfermeiros, técnicos de enfermagem, fisioterapeutas, etc.), voltada aos pacientes considerados críticos e de alta complexidade. Por essas características, nesse cenário predomina a oferta de cuidado realizado tecnicamente, e na grande maioria das vezes, a partir de um modelo de assistência biologicista, linear, pontual, fragmentado e mecanizado, sem cuidar das demais dimensões que integram o ser humano. Soma-se a essa realidade uma prática em detrimento de aspectos voltados ao relacionamento interpessoal e humanização da assistência $^{(6-7)}$.

O processo de trabalho nesse setor é permeado pela dor, sofrimento, morte, impotência, estresse laboral e insalubridade, de modo que é fundamental cuidar desses profissionais, dar-lhes condições de constituírem equipes capazes de promover a humanização do serviço em seu meio ambiente laboral (7-8), evitando-se a desumanização do próprio trabalhador.

Segundo alguns autores, ainda são incipientes as reflexões e estudos ${ }^{(8)}$ referentes ao tema (des)humanização dos serviços de saúde e sua interface com a saúde do trabalhador de enfermagem, sugerindo que essa tema deve ser mais investigado.

Diante do exposto, refletindo-se sobre a necessidade de novas investigações, surgiram os seguintes questionamentos de pesquisa: Quais fatores colaboram para que aconteça a humanização no processo laboral entre os trabalhadores de enfermagem que atuam em UTI? Quais fatores dificultam a ocorrência desse fenômeno entre esses profissionais?

Pondera-se que as respostas a esses questionamentos atendam o objetivo, proposto para este estudo, de identificar os fatores que propiciam e dificultam a humanização entre os trabalhadores de enfermagem, na percepção de enfermeiros de UTI.

Acredita-se que este estudo será de fundamental importância por contribuir para estimular os enfermeiros e as equipes de enfermagem na busca de estratégias que promovam a humanização no processo laboral entre os trabalhadores de enfermagem e, em 
consequência, nos cuidados aos pacientes.

\section{MÉTODO}

Estudo qualitativo-descritivo realizado com 10 enfermeiros que atuavam nos turnos matutino, vespertino e noturno de uma UTI de adulto em um Hospital Universitário localizado no estado do Paraná. O número de participantes foi definido pelo critério de saturação de dados, isto é, coletaram-se os dados até o momento em que ocorreu a convergência/repetição das falas ${ }^{(9)}$. Foi considerado critério de inclusão trabalhar na unidade há pelo menos um ano e não estar de férias e ou em licença no período de coleta dos dados.

A coleta de dados foi efetuada por meio de entrevistas semiestruturadas individuais realizadas no cenário do estudo, e aconteceu entre os meses de maio a julho de 2012, com duração aproximada de trinta minutos para cada entrevistado. O roteiro da entrevista continha as seguintes questões: Fale-me sobre quais os fatores que você julga que colaboram para que ocorra a humanização entre os profissionais de enfermagem em seu processo de trabalho? E quais fatores dificultam para que aconteça a humanização entre esses profissionais em seu processo de trabalho?

As informações obtidas nas entrevistas foram gravadas com a autorização dos participantes e, posteriormente, transcritas na íntegra $e$ submetidas à análise de conteúdo, na modalidade temática, seguindo-se três etapas: pré-análise, exploração do material e tratamento dos dados, inferência e interpretação. Durante a pré-análise, fez-se a leitura flutuante, organizando-se o material com o intuito de apreender as principais ideias e aspectos relevantes nele encontrados. Posteriormente, realizou-se a exploração do material, com agrupamento dos dados brutos em núcleos de compreensão do texto e codificação, isto é, a sistematização em categorias temáticas relacionadas às características do conteúdo. Por fim, procedeu-se ao tratamento dos resultados, inferência e interpretação, mediante articulação entre o material empírico estruturado e a literatura ${ }^{(9)}$.

A pesquisa foi conduzida de acordo com os padrões éticos preconizados pela Resolução 196/96 ${ }^{(10)}$ do Conselho Nacional de Saúde, sendo aprovada pelo Comitê de Ética em Pesquisa da Universidade Estadual de Londrina de acordo com o Parecer $\mathrm{n}^{\circ}$ 33421/11, conforme CAAE:
0300.0.268.000-11, e a anuência para participação na pesquisa foi obtida por meio da assinatura o Termo de Consentimento Livre e Esclarecido.

\section{RESULTADOS}

Em relação à caracterização dos enfermeiros que participaram do estudo, todos pertenciam ao sexo feminino, com idades entre 30 e 42 anos e atuavam na UTI de adultos há mais de oito anos.

A análise das entrevistas levou à construção de duas categorias temáticas: Fatores que propiciam, e fatores que dificultam a humanização laboral entre os trabalhadores de enfermagem.

\section{Fatores que propiciam a humanização laboral entre os trabalhadores de enfermagem}

Nessa categoria apreendeu-se que o trabalho desenvolvido em equipe e o bom relacionamento interpessoal são fatores que colaboram para que ocorra a humanização entre os trabalhadores, conforme se identifica nas falas a seguir:

Para mim o trabalho em equipe e o bom relacionamento entre nós é uma das formas de conseguirmos a humanização entre os profissionais. (E4)

Humanização entre os profissionais de enfermagem é atuarmos em equipe e desenvolvermos boas relações entre nós diariamente. [...] uma relação de respeito e empatia. (E5)

Um ambiente onde haja a união, cooperação e trabalho realizado em equipe, bem como um ótimo relacionamento interpessoal para que possamos estabelecer um processo laboral humanizado. (E9)

Outros fatores que facilitam a humanização, na concepção das enfermeiras, estão relacionados à valorização do outro e de si próprio, envolvendo nesse processo tanto os colegas quanto a instituição. Esse resultado pode ser observado nos seguintes fragmentos:

Humanização é valorizar o outro, o que o outro tem de melhor, ver as qualidades que cada um possui para que o trabalho seja o mais humano possível. $\hat{E}$ preciso também que a instituição nos valorize. (E6)

Quando a gente valoriza e é valorizada a humanização aflora entre a equipe e o ambiente de trabalho fica muito leve. (E7)

A nossa prática cotidiana é vivenciada por problemas reais, e só vamos conseguir solucionar se nos ajudarmos mutuamente, com bases nos valores da humanização para buscar as possíveis saídas. [...]. 
A humanização entre os trabalhadores se constitui em uma das ferramentas essenciais para que $o$ processo de trabalho torne-se humanizado, e por consequência, para a assistência prestada ao cliente. (E8)

A comunicação configurou-se uma ferramenta indispensável para haver humanização entre os enfermeiros em seu processo de trabalho, o que pode ser constatado no seguinte discurso:

No meu entender a comunicação é uma forma de conseguir a humanização entre os colegas. Ouvir e ser ouvido, respeitar o outro, ser respeitado, acredito que torna nossas relações mais humanas, mas isso a gente consegue colocar em prática com a experiência dos anos trabalhados. (E10)

Os valores pessoais, segundo as entrevistadas, são essenciais para que aconteça a humanização entre os trabalhadores. $\mathrm{Na}$ concepção das enfermeiras, essa característica é inata, mas pode também ser aprimorada durante a formação, conforme mostram as falas a seguir:

A humanização entre os trabalhadores é algo inerente a cada pessoa, a pessoa já nasce com essa visão, é uma questão de educação e principalmente de significado para cada um, isto é dos valores pessoais. (E3)

Temos que por em prática a humanização para que possamos ter um trabalho menos desgastante e para isso é preciso ter e seguir uma conduta, [...] é colocar na prática o que acreditamos, isto é a nossa concepção. Porém, destaco a importância de aprendermos em nossa formação como consolidarmos a humanização entre os profissionais no cotidiano laboral, pois o destaque é sempre para a humanização nos cuidados aos pacientes. (E5)

\section{Fatores que dificultam a humanização laboral entre os profissionais de enfermagem}

Nessa categoria, os depoimentos das entrevistadas revelam que a falta de reconhecimento e $\mathrm{O}$ individualismo são fatores que dificultam a humanização entre os trabalhadores de enfermagem, conforme se evidencia nos seguintes discursos:

Há pessoas que são muito individualistas e não reconhecem o que nós fazemos isso na minha visão são fatores que levam à falta de humanização no ambiente laboral, pois fazemos parte de uma equipe e o individualismo não ajuda em nada, mas sim atrapalha os laços de humanização entre os trabalhadores no cotidiano laboral. (E2)

A falta de reconhecimento "mina" qualquer chance de humanização entre os membros da equipe de trabalho quando não somos reconhecidas não dá vontade de fazer nada para melhorar o nosso processo de trabalho. (E6)

A nossa prática cotidiana é vivenciada por problemas reais, e só vamos conseguir solucionar se nos ajudarmos mutuamente, se predominar a individualidade não avançamos para a humanização entre os trabalhadores de enfermagem e nem tampouco para os cuidados aos pacientes. (E8)

\section{DISCUSSÃO}

No entendimento das participantes deste estudo, a humanização entre os profissionais de enfermagem seiniciacom umbom relacionamento interpessoal desses trabalhadores e no trabalho em equipe.

Alguns autores ${ }^{(11-12)}$ afirmam que as relações interpessoais no trabalho, tanto entre a equipe de enfermagem quanto entre a multiprofissional, quando não se desenvolvem satisfatoriamente, produzem um ambiente de trabalho desfavorável que interfere em todo o processo laboral, gera insatisfação no profissional, e, em consequência, dificulta a humanização entre os trabalhadores.

O bom relacionamento em equipe está diretamente ligado à empatia, ou seja, quando o trabalhador compreende o outro, torna o relacionamento mais espontâneo e efetivo, estabelece atitudes humanizadas entre seus pares, além de desenvolver uma consciência sobre o seu papel no grupo, em uma dinâmica interdependente e complementar, pois trabalhador e grupo visam aos mesmos objetivos $^{(13)}$.

Pesquisa ${ }^{(14)}$ que investigou fatores de insatisfação de trabalhadores de uma UTI revelou que o trabalho desenvolvido em equipe é mais satisfatório quando envolve o compartilhamento de informações. Assim, o trabalho em equipe favorece e proporciona o crescimento de seus membros, incentivando-os a buscar conhecimentos e habilidades que outrora não possuíam, e incentiva a cooperação mútua ${ }^{(15)}$.

Essa troca de saberes possibilitada pelo trabalho em equipe é considerada pela $\mathrm{PNH}$ uma das formas de promover a humanização nos processos de trabalho $^{(3)}$. E deve ser valorizada especialmente no ambiente de UTI, que se caracteriza por ser um cenário de inovação e atendimento especializado de enfermagem, potencializado pela necessidade de constante desenvolvimento de competências 
dos profissionais, a fim de harmonizar o serviço entre a alta tecnologia e a assistência ${ }^{(6)}$.

Neste estudo, as enfermeiras evidenciaram que a valorização do trabalhador por si próprio, por seus colegas e pela instituição também se constitui em mecanismo que propicia a humanização no processo de trabalho desses profissionais.

Humanizar o ambiente de trabalho também implica interagir com o colega de modo acolhedor, valorizando seus saberes, habilidades e competências, olhando o outro em suas fragilidades e necessidades, sob as lentes da ética e da solidariedade, e apostando em redes interdisciplinares de relacionamento profissional. Assim, a valorização profissional deve ser um princípio fundamental na gestão de pessoas, a fim de que colaboradores sintam-se motivados e reconhecidos para desenvolver o seu trabalho com satisfação $\mathrm{O}^{(8)}$. Portanto, pode-se afirmar que, para manter um bom ambiente de trabalho ou um ambiente humanizado, é necessário compreender que a pessoa é um ser único, individual e capaz de reagir de diferentes formas diante das relações de trabalho, as quais auxiliam a construção de ambientes saudáveis.

Autores $^{(5,16)}$ refletem sobre a questão de cuidar do cuidador, isto é, como cuidar e valorizar a pessoa que trabalha, e afirmam que existem dois processos essenciais no processo laboral que envolve o profissional e determinam sua valorização e satisfação no trabalho: o desejo de reconhecimento; e o reconhecimento do desejo. O primeiro envolve remuneração, perspectivas de crescimento e reconhecimento, formas de vínculo empregatício e acesso a tecnologias de cuidado que propiciem a produção de saúde pelo trabalhador como recurso para a dignificação de seu labor e ampliação de seu valor de uso. $O$ segundo relaciona-se à expressão de suas peculiaridades, do que os faz únicos. Na prática, isso ocorre pela efetiva inclusão das pessoas nos processos de tomada de decisão nas organizações.

Outro estudo também mostrou que o reconhecimento profissional é essencial para que ocorra a humanização do processo de trabalho dos enfermeiros e demais trabalhadores de enfermagem ${ }^{(17)}$.

A comunicação foi evidenciada neste estudo como um dos alicerces na humanização do processo de trabalho corroborando o que preconiza a $\mathrm{PNH}^{(3)}$, pois humanizar significa dar o espaço que a comunicação demanda, ou seja, é preciso que o sentimento humano e as percepções verbalizadas pelo sujeito sejam reconhecidas pelo outro. Assim, a humanização é dependente da capacidade de falar e de ouvir: sem comunicação não há humanização. Os trabalhadores de enfermagem precisam estar dispostos e envolvidos para estabelecer essa relação, por meio de uma comunicação efetiva ${ }^{(18)}$. Mesmo que esses últimos autores mencionados insiram uma perspectiva centrada nas relações de cuidado ao usuário, acredita-se que isso também se aplique ao cuidado ao profissional, pois ambos são sujeitos protagonistas do processo de produção de saúde.

Em relação ao preparo na formação para a humanização entre os profissionais, é fato que, na grande maioria dos currículos de enfermagem, ainda não há um equilíbrio entre a formação que prima pela humanização nos cuidados ao usuário e a dispensada ao profissional. Assim sendo, é preciso que seja revisto esse fato nos currículos de enfermagem ${ }^{(19-20)}$.

Ainda no presente estudo, evidenciou-se que a falta de reconhecimento e o individualismo são fatores que dificultam o estabelecimento da humanização entre os trabalhadores no processo de trabalho. Isto porque o trabalho de enfermagem está inserido no modelo capitalista, no qual a polivalência, a flexibilidade, os resultados e a exigência de altos níveis de desempenho são elementos que tendem a não valorizar, ou seja, a não reconhecer a contribuição desse trabalhador, o que provoca impactos sobre a saúde deles ${ }^{(21)}$.

Outros autores ${ }^{(22-23)}$ afirmam que a falta de reconhecimento na enfermagem é histórica e se mantém na atualidade, trazendo consigo um sentimento de desvalorização, além de repercutir intensamente em suas vivências de sofrimento. Recomendam que o reconhecimento do trabalho deva ser incorporado no próprio processo laboral, por meio de palavras, expressões, gestos ou nos momentos de avaliação formal ${ }^{(23)}$.

A ausência de reconhecimento, o individualismo e o isolamento são fatores que podem provocar insatisfação, desânimo, sofrimento, isolamento, agravos à saúde mental e mais drasticamente terminar em suicídio ${ }^{(24)}$.

Sabe-se que existem diferenças entre os valores de cada indivíduo e as exigências do meio, as quais podem ser observadas principalmente nas influências que as demandas sociais exercem sobre as pessoas. Assim sendo, os trabalhadores precisam utilizar estratégias de defesa para lidar 
com situações contraditórias e que não estão de acordo com a sua concepção para que a humanização prevaleça no ambiente laboral ${ }^{(25)}$.

\section{CONSIDERAÇÕES FINAIS}

Para os enfermeiros, os fatores que contribuem para a humanização no processo de trabalho entre os profissionais de enfermagem de UTI estão relacionados: ao trabalho realizado em equipe, ao bom relacionamento interpessoal, à valorização pelos colegas, pela instituição e pelo próprio trabalhador, à comunicação efetiva, aos valores de cada pessoa, e à formação acadêmica que deveria primar não somente pela humanização da assistência, mas também entre os profissionais.

Neste estudo, revelou-se também que a falta de reconhecimento pelo trabalho desenvolvido e as atitudes de individualismo são elementos que dificultam a concretização de relações positivas e que se estabeleça a humanização entre os trabalhadores.

Enfatiza-se que o objetivo do estudo foi atingido, porém, a humanização no processo de trabalho do enfermeiro é objeto de investigação que abarca a individualidade e, por sua vez, a concepção de cada pessoa. Devido a esses fatores e por retratar a realidade dos profissionais que trabalham em uma UTI de adultos de um Hospital Universitário, é necessário considerar os resultados em sua singularidade. Sugere-se a realização de novas pesquisas sobre o tema.

\section{REFERÊNCIAS}

1. Cardoso GB, Silva ALA. O processo de trabalho na enfermagem: articulação das tecnologias do cuidado. Rev. Enferm. UERJ. [Internet] 2010; 18(3) [acesso em 20 jun 2015]. Disponível: http://www.facenf.uerj.br/ v18n3/v18n3a20.pdf

2. Alves CA, Deslandes SF, Mitre RMA. A gestão do processo de trabalho da enfermagem em uma enfermaria pediátrica de média e alta complexidade: uma discussão sobre cogestão e humanização. Interface,Comum.,Saúde,Educ. [Internet] 2011; 15(37) [acesso em 26 maio 2015]. Disponível: http://dx.doi. org/10.1590/S1414-32832011005000008

3. Ministério da Saúde (BR). Secretaria de Atenção à Saúde. Núcleo Técnico da Política Nacional de Humanização. Humaniza SUS: cadernos de textos: cartilhas da política nacional de humanização. [Internet] Brasília (DF): Ministério da Saúde; 2011 [acesso em jun 20 2015]. Disponível: http://bvsms.saude.gov.br/ bvs/publicacoes/caderno_textos_cartilhas_politica humanizacao.pdf
4. Chernicharo IM, Silva FD, Ferreira MA. Caracterização do termo humanização na assistência por profissionais de enfermagem. Esc. Anna Nery. [Internet] 2014; 18(1) [acesso em 20 mar 2015]. Disponível: http://dx.doi. org/10.5935/1414-8145.20140023

5. Pasche DF, Passos E, Hennington EA. Cinco anos da política nacional de humanização: trajetória de uma política pública. Ciênc.Saúde Coletiva. [Internet] 2011; 16(11) [acesso em 18 abr 2015]. Disponível: http:// dx.doi.org/10.1590/S1413-81232011001200027

6. Camelo SHH, Santos VLS, Laus AM, Chaves LDP. Perfil profissional de enfermeiros atuantes em unidades de terapia intensiva de um hospital de ensino. Cienc.Enferm. [Internet] 2013; 19(3) [acesso em 23 mar 2015]. Disponível: http://dx.doi.org/10.4067/ S0717-95532013000300006

7. Backes MTS, Erdmann AL, Büscher A, Backes DS. O cuidado intensivo oferecido ao paciente no ambiente de Unidade de Terapia Intensiva. Esc. Anna Nery. [Internet] 2012; 16(4) [acesso em 20 mar 2015]. Disponível: http://dx.doi.org/10.1590/S141481452012000400007

8. Rosa CMR, Fontana RT. A percepção de técnicos em enfermagem de uma unidade de terapia intensiva sobre a humanização no seu trabalho. Cienc.Cuid. Saúde. [Internet] 2010; 9(4) [acesso em 20 abr 2015]. Disponível: http://dx.doi.org/10.4025/cienccuidsaude. v9i4.6654

9. Bardin L. Análise de conteúdo. Lisboa: Edições 70; 2011.

10.Ministério da Saúde (BR). Conselho Nacional de Saúde. Diretrizes e normas regulamentadoras de pesquisa envolvendo seres humanos. Resolução n. 196, de 10 de outubro de 1996. Brasília; 1996.

11. Jeong DJY, Kurcgant P. Fatores de insatisfação no trabalho segundo a percepção de enfermeiros de um hospital universitário. Rev Gaúcha Enferm. [Internet] 2010; 31(4) [acesso em 20 jun 2015]. Disponível: http:// dx.doi.org/10.1590/S1983-14472010000400007

12.Fontana RT. Humanização no processo de trabalho em enfermagem: uma reflexão. Rev. Rene. [Internet] 2010; 11(1) [acesso em 20 jun 2015]. Disponível: http:// www.revistarene.ufc.br/revista/index.php/revista/ article/view/364/pdf

13. Araújo MMT, Silva MJP. Estratégias de comunicação utilizadas por profissionais de saúde na atenção à pacientes sob cuidados paliativos. Rev. Esc.Enferm. USP. [Internet] 2012; 46(3) [acesso em 20 maio 2015]. Disponível: http://dx.doi.org/10.1590/S008062342012000300014

14. Pol P, Zarpellon LD, Matia G. Fatores de (in) satisfação no trabalho da equipe de enfermagem em 
UTI pediátrica. Cogitare Enferm. [Internet] 2014; 19(1) [acesso em 10 mar 2015]. Disponível: http://dx.doi. org/10.5380/ce.v19i1.35958

15. Navarro ASS, Guimaraes RLS, Garanhani ML. Trabalho em equipe: o significado atribuído por profissionais da estratégia de saúde da família. Reme, Rev. Min.Enferm. [Internet] 2013; 17(1) [acesso em 20 mar 2015]. Disponível: http://www.reme.org.br/artigo/ detalhes/579

16. Nunes CM, Tronchin DMR, Melleiro MM, Kurcgant P. Satisfação e insatisfação no trabalho na percepção de enfermeiros de um hospital universitário. Rev. Eletr. Enf. [Internet] 2010; 12(2) [acesso em 20 jun 2015]. Disponível: http://www.fen.ufg.br/revista/v12/n2/ v12n2a04.htm

17. Sprandel LIS, Vaghetti HH. Valorização e motivação de enfermeiros na perspectiva da humanização do trabalho nos hospitais. Rev. Eletr. Enf.[Internet] 2012; 14(4) [acesso em 25 maio 2015]. Disponível: http:// dx.doi.org/10.5216/ree.v14i4.16100

18. Broca PV, Ferreira MA. Equipe de enfermagem e comunicação: contribuiçõos para o cuidado de enfermagem. Rev.bras.enferm. [Internet] 2012; 65(1) [acesso em 23 maio 2015]. Disponível: http://dx.doi. org/10.1590/S0034-71672012000100014

19. Casate JC, Corrêa AK. A humanização do cuidado na formação dos profissionais de saúde nos cursos de graduação. Rev.esc. enferm. USP. [Internet] 2012; 46(1) [acesso em 23 maio 2015]. Disponível: http://dx.doi. org/10.1590/S0080-62342012000100029

20. Abrahão AL, Merhy EE. Formação em saúde e micropolítica: sobre conceitos-ferramentas na prática de ensinar. Interface, Comum., Saúde, Educ.[Internet] 2014; 18(49) [acesso em 20 maio 2015]. Disponível: http://dx.doi.org/10.1590/1807-57622013.0166

21. Traesel ES, Merlo ARC. Trabalho imaterial no contexto da enfermagem hospitalar: vivências coletivas dos trabalhadores na perspectiva da Psicodinâmica do Trabalho. Rev.bras.Saúde Ocup. [Internet] 2011; 36(123) [acesso em 29 abr 2015]. Disponível: http://www.scielo. br/pdf/rbso/v36n123/a05v36n123.pdf

22. Campos JF, David HMSL, Souza NVDO. Prazer e sofrimento: avaliação de enfermeiros intensivistas à luz da psicodinâmica do trabalho. Esc. Anna Nery. [Internet] 2014; 18(1) [acesso em 20 jun 2015]. Disponível: http:// dx.doi.org/10.5935/1414-8145.20140013

23. Garcia AB, Dellaroza MSG, Haddad MCL, Pachemshy LR. Prazer no trabalho de técnicos de enfermagem do pronto-socorro de um hospital universitário público. Rev. Gauch. Enferm. [Internet] 2012; 33(2) [acesso em 20 jun 2015]. Disponível:http://dx.doi.org/10.1590/ S1983-14472012000200022
24. Dejours C, Bègue F. Suicídio e trabalho: o que fazer? Brasília: Paralelo 15; 2010.

25. Meneghini F, Paz AA, Lautert L. Fatores ocupacionais associados aos componentes da Síndrome de Burnout em trabalhadores de enfermagem. Texto Contexto Enferm. [Internet] 2011; 20(2) [acesso em 20 mar 2015]. Disponível: http://dx.doi.org/10.1590/S010407072011000200002 